\title{
$f(R)$ Gravitasyon Teorisinde Toz Bulutlu Konformal Düz Uzay-Zamanlar
}

\author{
Doğukan Taşer \\ Çanakkale Onsekiz Mart Üniversitesi, Çan Meslek Yüksekokulu, Elektrik ve Enerji Bölümü, 17400, Çanakkale, \\ Türkiye \\ (ORCID: https://orcid.org/0000-0002-8622-6830)
}

(Alınıș / Received: 11.07.2018, Kabul / Accepted: 29.01.2019, Online Yayınlanma / Published Online: 17.04.2019)

\section{Anahtar Kelimeler}

$\mathrm{f}(\mathrm{R})$ gravitasyon teorisi,

Toz madde,

Konformal uzay-zaman

\begin{abstract}
Özet: Bu çalışmada, toz bulutu bulunan konformal düz uzay-zamanlar $f(R)$ gravitasyon teorisi çerçevesinde incelenmiştir. Toz bulutu varlı̆ı̆nda konformal düz uzay-zamanların alan denklemleri ve tam çözümleri herhangi bir yaklaşım ve kısıtlayıcı metot kullanılmadan elde edilmiștir. $f(R)$ gravitasyon teorisi çerçevesinde konformal düz uzay-zamanların sabit enerji yoğunluklu toz bulutu varlığına izin verdiği gösterilmiștir. Teorinin dinamik yapısını karakterize eden $f(R)$ fonksiyonu oluşturulan model için alan denklemlerinin çözümlerinden elde edilmiştir. Oluşturulan model için elde edilen $f(R)$ fonksiyonu ile teoride farklı koşul ve durumları ifade etmek için kullanılan uygulanabilir $f(R)$ fonksiyonları karşılaștırılmıştır. Metrik potansiyelin radyal koordinata göre değişimi grafik yardımıyla irdelenmiş ve çözümlerin sınır durumları incelenmiştir. Son olarak, elde edilen sonuçlar fiziksel ve geometrik açıdan irdelenerek tartışılmıştır.
\end{abstract}

\section{Conformally Flat Space-Time with Dust Matter in $f(R)$ Gravity}

\section{Keywords}

$f(R)$ gravitation theory, Dust matter, Conformal space-time

\begin{abstract}
In this study, conformally flat space-times filled with dust matter is investigated in framework of $f(R)$ gravitation theory. Exact solutions and field equations of conformally flat space-time in the presence of dust matter are obtained without using any restrictive method and approximation. It is shown that conformally flat space-times in framework of $f(R)$ gravitation theory permit to dust matter with constant energy density. $f(R)$ function which characterise dynamic structure of theory is obtained from solutions of field equations for constructed model. Obtained $f(R)$ function for constructed model and viable $f(R)$ functions, which are used to express various conditions and situations in theory, are compared. Change of the metric potential with respect to radial coordinate is examined by the help of graphic and limiting cases of the solutions are investigated. Finally, obtained results are discussed in the terms of physical and geometric aspects.
\end{abstract}

\section{Giriş}

1990’ların başından itibaren, teknolojideki büyük sıçrayış ile paralel olarak gözlemsel kozmolojideki gelişmeler de büyük bir hız ile artış göstermiștir. Gözlemsel kozmolojide gerçekleșen bu gelișmeler ile bağlantılı olarak, evrenin evrimi süresince göstermiş olduğu davranış hakkındaki bilgilerimiz de değişime uğramıştır. Süpernova Tip-Ia gözlemleri [1,2,3], kozmik mikrodalga fon ışınımı deneyleri [4,5], X-ışını deneyleri [6] ve büyük ölçekli yapı ölçümleri [7] gibi gözlemsel deneyler ve ölçümler günümüz evreninin artan bir ivme ile genişlediğine işaret etmektedir [8]. $\mathrm{Bu}$ nedenle, evrenin ivmeli olarak genişlemesinin ardındaki itmeye neden olan mekanizma kozmolojik açından en çok efor sarf edilen ve teorik olarak tatmin edici bir biçimde ifade edilemeyen en popüler konulardan bir tanesidir. Kozmolojik açıdan bakıldığında, günümüz evreninin artan bir ivme ile genişlemesini açıklamak için teorik olarak iki farklı tutum sergilenmektedir. Bunlardan ilki Genel Relativite alan denklemlerinde itici etkiye sahip bir egzotik madde formu tanımlamaktır. Bu egzotik madde formu genel olarak "karanlık enerji" olarak adlandırılır [9]. Kozmik mikrodalga fon ışınımı deneyleri ve Süpernova gözlemleri bilinen evrenin \%76 oranında karanlık enerjiden, \%21 oranında karanlık maddeden ve \%4 oranında baryonik maddeden oluştuğuna işaret etmektedir [9]. Literatürde, evrenin artan bir ivme ile genişlemesini 
açıklamak ve karanlık enerjinin yapısını ve kaynağını keşfetmek için quintessence madde [10], hayalet enerji [11], takyon [12], k-essence madde [13] ve Chaplygin gaz [14] gibi kozmolojik karanlık enerji modelleri önerilerek detaylı olarak çalışılmıştır. Bununla birlikte, alan denklemlerinde karanlık enerjiyi simgelemek için kullanılan diğer bir yaklaşım ise durum denklemi (EoS) veya kozmolojik sabit kullanımıdır [15]. Bütün bu kozmolojik öneri ve modellemelere rağmen evrendeki bu dominant karakterin kökeni ve çalışma mekanizması gizemini koruyan konuların bașında gelmektedir. Günümüz evreninin davranışını ifade etmek için kullanılan ikinci tutum ise Genel Relativite teorisinin geometri kısminın modifiye edilmesidir. Genel Relativite teorisinin geometri kısmının modifikasyonları, herhangi bir karanlık enerji modeline ihtiyaç duymadan evrenin bugünkü ivmeli genişlemesini açıklamak ve evrenin dinamik yapısını anlamak için başarılı bir yöntem olarak düşünülmektedir. Bu nedenle son zamanlarda çok fazla ilgi görmektedir. Modifiye teoriler Einstein-Hilbert eyleminde Ricci skalerinin yerine keyfi bir fonksiyonun tanımlanmasıyla elde edilir. Son zamanlarda popüler olarak kullanilan modifiye gravitasyon teorileri $f(R)$ teori [16], $f(T)$ teori [17], $f(R, T)$ teori [18] ve $f(G)$ teori [19] olarak siralanabilir.

Yukarıda bahsedildiği gibi, Genel Relativite teorisinin bilinen en popüler modifikasyonlarından bir tanesi $f(R)$ gravitasyon teorisidir. Genel Relativite teorisinde, Einstein-Hilbert etkisinde geometrik kısmın Lagrangian yoğunluğu Ricci skaleri ile tanımlanırken, $f(R)$ gravitasyon teorisinde geometrik kısmın Lagrangian yoğunluğu Ricci skalerine bağlı lineer bir fonksiyon olan $f(R)$ fonksiyonu ile tanımlanır [18]. $f(R)$ gravitasyon teorisi kendi içinde birçok uygulanabilir $f(R)$ fonksiyonu içermektedir. Bu nedenle evrenin evrimi sırasında gösterdiği dönemler uygulanabilir $f(R)$ fonksiyonları aracılığıyla tek bir teori altında incelenebilir. Nojiri ve Odintsov (2007) [20] evrenin geçiş dönemi olarak adlandırılan ve evrenin genişleme hızındaki artışın farklılık gösterdiği bu dönemde, $f(R)$ gravitasyon teorisinin evrenin genişleme hızının zamanla küçülerek artığını daha sonrasında ise genişleme hızının büyüyerek arttığını göstermişlerdir. Nojiri ve Odintsov (2008) [21] farklı uygulanabilir $f(R)$ fonksiyonları aracılığıyla teorinin evrenin enflasyon dönemi ile günümüz ivmeli genişlemesinin bir arada açıklanmasına uygun olduğunu göstermişlerdir. Bu özelliklerinin yanı sıra, $f(R)$ gravitasyon teorisi karanlık enerjiye alternatif bir gravitasyonel etkiye alan denklemlerinde sahip olduğu için dikkatleri üzerine çeken bir teoridir. Bu kapsamda, Carroll ve ark. [22] evrenin günümüz ivmeli genişlemesini herhangi bir itici madde formuna ihtiyaç duymadan $f(R)$ gravitasyon teorisinde açıklamıştır. Ayrıca, $f(R)$ gravitasyon teorisi yüksek enerji fiziğinde de, Hiyerarşi Probleminin incelenmesi, gravitasyon ile Büyük Birleşim Kuramının birleşimi, gravitasyon dalgalarının tespiti ve tekillik problemi gibi bazı ilgi çeken konuların araştırılmasında da dikkate alınmaktadır [23,24].

Genel relativite teorisinde uzay-zamanı ifade etmek için kullanılan $g^{i k}$ metrik potansiyeli 4-boyutlu uzayzamanda simetri özelliğinden dolayı on tane bileşenden oluşmaktadır. Herhangi bir kısıtlama yapmadan fiziksel olarak anlamlı çözümler bulmak oldukça önemlidir. Bu nedenle belirli gravitasyonel alanlar için konformal olan uzay-zaman modellerinin fiziksel özelliklerinin araştırılması büyük bir ilgi görmektedir [25]. Bunlardan bir tanesi de konformal düz uzay-zamanlardır. Konformal düz uzayzamanlarda lineer bağımsız metrik potansiyeli sayısı bire düşmektedir. Schwarzschild iç çözümü ve Lemâitre kozmolojik evreni gibi fiziksel olarak önemli birçok konformal düz uzay-zaman modeli bulunmaktadır [25]. Reddy [26] statik küresel simetrik konformal düz uzay-zamanı Brans-Dicke ve Sen-Dunn gravitasyon teorilerinde incelemiştir. Her iki teori içinde düz bir uzay-zaman tanımlayan vakum çözümleri elde etmişlerdir. Reddy ve ark. [27] vakum durumunda konformal düz küresel simetrik statik uzay-zamanı Self-Creation Kozmolojide incelemiştir. Elde ettikleri çözümleri Brans-Dicke teorideki çözümler ile karşılaștırarak farklılıklarını göstermişlerdir. Yadav ve Prasad [28] ideal akışkan varlığında statik olmayan konformal düz uzay-zamanı Einstein-Cartan teori kapsamında incelemişlerdir. Elde edilen çözümlerin kinematik özelliklerini araştırmışlardır. Khadekar ve Nagpure [29] ideal akışkan varlığında yüksek boyutlu statik konformal düz uzay-zamanı Lyra teoride incelemiştir. Elde edilen çözümlerin Einstein-Cartan teoride yapılan çözümlerle benzerliğini tartışmışlardır. Pradhan ve Pandey [25] statik konformal düz uzay-zamanı kozmolojik sabitli Einstein alan denklemlerini hem yüklü ideal akışkan hem de bulk vizkoz akışkan varlığında incelemiştir. Her iki çözüm için de, kozmolojik sabitin hem pozitif hem de zamanla azalan bir fonksiyon olduğunu göstermişlerdir. Abebe ve ark. [30] konformal düz ışın yayan yıldızları Lie simetri kullanarak araştırmışlardır. Sınır koşulları için yeni bir tam çözüm sınıfı elde etmişleridir. Evriminin şu anki sürecinde evren küresel simetriktir ve madde dağılımı homojen ve izotropiktir. Birçok kozmolojik model tanımlanırken evrenin madde dağılımı ideal akışkan veya basınçsız bir dağılım olan toz madde ile tanımlanır. Sharif ve Kausar [31] $f(R)$ gravitasyon teorisi çerçevesinde küresel simetrik toz bulutu alan denklemlerini ve Landau-Lifshitz enerjimomentum dağılımını incelemiştir. Ricci eğrilik skalerinin sabit olduğu durumda alan denklemlerinin çözümlerini elde etmişlerdir. Shamir ve Raza [32] silindirik uzay-zamanda toz bulutu dağılımını $f(R)$ teori kapsamında incelemişlerdir. Yaptıkları çalışmada Ricci eğrilik skalerinin sabit olduğu durumda toz maddenin enerji yoğunluğunun sabit olduğunu, Ricci eğrilik skalerinin sabit olmadığı durumda toz maddenin enerji yoğunluğunun radyal 
koordinata bağlı olarak farklı seçimler altında azalan veya artan bir fonksiyon olduğunu göstermişlerdir. Shamir ve ark. [33] $f(R)$ gravitasyon teorisinde toz maddenin gravitasyonel çöküşünü incelemişlerdir. Yıldızın iç bölgesinde Friedmann-Robertson-Walker metriğini göz önünde bulundururken, yıldızın dış bölgesinde Schwarzchild metriğini dikkate almışlardır. Sabit eğrilik skaleri durumunda çözümler elde etmişler ve belirli koşullar kullanarak gravitasyonel kütleyi bulmuşlardır.

Bilindiği gibi alan denklemlerinin tam çözümü madde ve geometri arasındaki ilişkiyi anlamak için oldukça önemlidir. Bu bağlamda, $f(R)$ gravitasyon teorisinde herhangi bir yaklaşım veya kısıtlama kullanmadan alan denklemi çözümü elde etmek teoride lineer bir $f(R)$ fonksiyonu tanımlandığı için hem Genel Relativite teorisine göre daha zordur hem de daha değerlidir. $\mathrm{Bu}$ çalışmanın amacı $f(R)$ gravitasyon teorisinde konformal düz uzay-zamanlarda toz bulutu dağılımının geometrik ve fiziksel davranıșını herhangi bir kısıtlama kullanmaksızın elde etmektir. Ayrıca önerilen model için $f(R)$ fonksiyonunun yapısını elde ederek uygulanabilir $f(R)$ modelleri ile ilişkisinin araştırılmasıdır.

Bu çalışma aşağıdaki gibi düzenlenmiştir: 2. Bölümde $f(R)$ gravitasyon teorisinin alan denklemleri tekrar hatırlatılmıştır. 3. Bölümde, konformal düz uzayzamanlarda bulunan toz bulutunun alan denklemleri $f(R)$ gravitasyon teorisi çerçevesinde elde edilmiştir ve önerilen model için alan denklemlerinin tam çözümleri elde edilmiştir. 4. Bölümde ise modele ait fiziksel ve geometrik sonuçlar tartışılmıștır.

\section{Materyal ve Metot}

Genel Relativite teorisinin modifikasyonu olan $f(R)$ gravitasyon teorisinde, Ricci skaleri geometrik olarak Einstein-Hilbert eyleminde önemli bir role sahiptir. Teoride Einstein-Hilbert eylemi Ricci skalerinin lineer bir fonksiyonu olan, $f(R)$ fonksiyonu aracılığı ile karakterize edilir [16]. $f(R)$ gravitasyon teorisi için Einstein-Hilbert eylemi aşağıdaki gibi tanımlanır:

$$
S=S_{g}+S_{m}\left(g_{i k}, \psi\right)=\int \sqrt{-g} d^{4} x\left(\frac{f(R)}{2}+S_{m}\right)
$$

Burada $f(R)$, Ricci skalerine bağlı bir fonksiyondur. $S_{m}$ kozmik maddenin Lagrangian yoğunluğunu ifade eder. $\psi$ ise kozmik madde alanını ifade eder [16]. (1) denkleminin varyasyonu alınarak, $f(R)$ gravitasyon teorisinin alan denklemi aşağıdaki gibi elde edilir:

$$
f_{R} R_{i k}-\frac{1}{2} f g_{i k}-\left(\nabla_{i} \nabla_{k}-g_{i k} \square\right) f_{R}=T_{i k}^{(m)}
$$

Burada $f_{R} \equiv \frac{d f}{d R} \quad$ ve $\square$ simgesi d'Alambertian operatörüdür [16]. (2) denklemi ile verilen $f(R)$ gravitasyon teorisi alan denkleminin izi dikkate alınırsa,

$$
f_{R} R-2 f+3 \square f_{R}=T^{(m)} .
$$

elde edilir. Burada $T^{(m)}$ enerji-momentum tensörünün izidir. (3) denklemi $f$ ve $f_{R}$ fonksiyonları arasındaki ilişkiyi veren yeni bir kısıtlayıcı denklem olarak kullanılabilir. (2) ve (3) denklemleri birlikte kullanılarak, $f(R)$ gravitasyon teorisi alan denklemleri Genel Relativite alan denklemleri formuna benzer olarak aşağıdaki gibi yeniden formüle edilebilir:

$$
G_{i k} \equiv R_{i k}-\frac{1}{2} R g_{i k}=T_{i k}^{(e f t .)} .
$$

Burada $\mathrm{T}_{\mathrm{ik}}^{\text {(eft.) }}$ etkin enerji-momentum tensörüdür ve $\mathrm{T}_{\mathrm{ik}}^{(\text {eft.) }}=\mathrm{T}_{\mathrm{ik}}^{(\text {eğr. })}+\breve{\mathrm{T}}_{\mathrm{ik}}^{(\mathrm{m})}$ şeklinde tanımlanır [34]. $\breve{\mathrm{T}}_{\mathrm{ik}}^{(\mathrm{m})}$ ise

$$
\check{T}_{i k}^{(m)}=T_{i k}^{(m)} / f_{R}
$$

şeklinde elde edilir. $\mathrm{T}_{\mathrm{ik}}^{\text {(eğr.) }}$ ve $\mathrm{T}_{\mathrm{ik}}^{(\mathrm{m})}$ sırasıyla eğrilik enerji-momentum tensörü [34] ve stres enerjimomentum tensörü [34] olarak adlandırılır ve

$$
\begin{gathered}
T_{i k}^{(e \breve{\mathrm{g}} r .)}=\frac{1}{f_{R}}\left[\nabla_{i} \nabla_{k} f_{R}-\frac{1}{4} g_{i k}\left(f_{R} R+\square f_{R}+T\right)\right], \\
T_{i k}^{(m)}=-\frac{2}{\sqrt{-g}} \frac{\delta\left(\sqrt{-g} S_{m}\right)}{\delta\left(g^{i k}\right)}
\end{gathered}
$$

şeklinde elde edilir.

\section{Bulgular}

Konformal düz küresel simetrik uzay-zaman için yay elemanı aşağıdaki gibi ifade edilir [30]:

$$
d s^{2}=A^{2}\left(-d t^{2}+d r^{2}+r^{2} d \Omega^{2}\right) .
$$

Burada metrik potansiyel $A, r$ radial koordinatına bağlı bir fonksiyondur ve $d \Omega^{2}=d \theta^{2}+$ $\sin ^{2} \theta d \phi^{2}$ şeklinde ifade edilir [35]. Uzay-zamanın eğrilik özelliklerini ifade eden Ricci skaleri (8) denklemi ile verilen konformal düz küresel simetrik uzay-zaman için

$$
R=-\frac{6 A^{\prime \prime}}{A^{3}}-\frac{12 A^{\prime}}{A^{3} r}
$$

olarak elde edilir. Burada kesme işareti (') $r$ radyal koordinata göre kısmi türevi ifade etmektedir. (9) denkleminde görüldüğü üzere Ricci skaleri metrik potansiyeli de içeren radyal koordinata bağlı bir fonksiyondur. Ricci skalerine bağlı bir fonksiyon olan $f_{R}(R)$ fonksiyonu, Ricci skalerinin bağlı olduğu radial koordinatın bir fonksiyonu olarak incelenebilir 
$\left(f_{R}(R) \approx f_{R}(r)\right)$. Toz bulutu için stres enerjimomentum tensörü ise

$$
T_{i k}^{(m)}=\rho u_{i} u_{k}
$$

şeklinde tanımlanır [31]. Burada $\rho$ toz bulutunun enerji yoğunluğudur ve $u_{i}$ co-moving koordinatlarda 4-lü hız vektörüdür [31].

(4)-(10) denklemlerini kullanarak, $f(R)$ gravitasyon teorisi çerçevesinde konformal düz uzay-zamanda bulunan toz bulutu için alan denklemleri aşağıdaki gibi elde edilir:

$$
\begin{aligned}
& \left(\frac{A^{\prime}}{A^{3}}+\frac{F^{\prime}}{2 A^{2} F}\right)\left(\frac{3 A^{\prime}}{A}+\frac{1}{r}\right)-\frac{3 A^{\prime \prime}}{2 A^{3}}-\frac{3 F^{\prime \prime}}{4 A^{2} F}-\frac{\rho}{4 F}=0, \\
& \left(-\frac{A^{\prime}}{A^{3}}-\frac{F^{\prime}}{2 A^{2} F}\right)\left(\frac{A^{\prime}}{A}+\frac{1}{r}\right)+\frac{A^{\prime \prime}}{2 A^{3}}+\frac{F^{\prime \prime}}{4 A^{2} F}-\frac{\rho}{4 F}=0
\end{aligned}
$$

ve

$$
\left(\frac{A^{\prime}}{A^{3}}+\frac{F^{\prime}}{2 A^{2} F}\right)\left(-\frac{A^{\prime}}{A}+\frac{1}{r}\right)+\frac{A^{\prime \prime}}{2 A^{3}}+\frac{F^{\prime \prime}}{4 A^{2} F}+\frac{3 \rho}{4 F}=0 .
$$

(11)-(13) denklemlerinden görüldüğü üzere, alan denklemleri üç bilinmeyenli adi diferansiyel denklem sistemi şeklinde elde edilir. Alan denklemi çalışmalarında hiçbir yaklaşım ya da kısıtlama kullanmadan tam çözüm elde edilmesi oldukça önemlidir. (11)-(13) ile verilen adi diferansiyel denklem sistemi kullanılarak konformal düz uzayzamanda bulunan toz bulutu için metrik potansiyeli, $f_{R}$ fonksiyonu ve toz bulutunun enerji yoğunluğu denklem sisteminin tam çözümünden sırasıyla

$$
\begin{aligned}
& A(r)=-\frac{2}{c_{1} r^{2}+2 c_{2}}, \\
& f_{R}(r)=-\frac{2 c_{3}}{c_{1} r^{2}+2 c_{2}},
\end{aligned}
$$

ve

$$
\rho=-3 c_{1} c_{3}
$$

şeklinde elde edilir. (16) denkleminden görüldüğü üzere $f(R)$ gravitasyon teorisi çerçevesinde konformal olarak uzay-zamanda bulunan toz bulutunun enerji yoğunluğu sabit bir fonksiyondur. (8) ve (14) denklemleri birlikte kullanılarak, $f(R)$ gravitasyon teorisi çerçevesinde konformal düz uzay-zaman için yay elemanı

$$
d s^{2}=\left(-\frac{2}{c_{1} r^{2}+2 c_{2}}\right)^{2}\left(-d t^{2}+d r^{2}+r^{2} d \Omega^{2}\right)
$$

şeklinde yeniden yazlabilir. (9) ve (14) denklemleri dikkate alınarak, toz bulutu varlığında konformal düz uzay-zaman için Ricci skaleri

$$
R(r)=-3 c_{1}\left(c_{1} r^{2}-3 c_{2}\right)
$$

olarak elde edilir. (18) denkleminde görüldüğü üzere oluşturulan modelin eğrilik özelliklerini ifade eden Ricci skaleri radyal koordinata bağlı bir fonksiyondur. (18) denkleminden faydalanılarak $r$ terimi Ricci skalerine bağlı bir fonksiyon olarak aşağıdaki gibi

$$
r(R)= \pm \frac{\sqrt{27 c_{1} c_{2}-3 R}}{3 c_{1}}
$$

tanımlanabilir. (15) ve (19) denklemleri dikkate alınarak, Ricci skalerine bağlı $f_{R}(R)$ fonksiyonu

$$
f_{R}(R)=\frac{6 c_{1} c_{3}}{R-12 c_{1} c_{2}}
$$

şeklinde elde edilir. (20) denkleminden faydalanılarak ve $f_{R} \equiv \frac{d f}{d R}$ tanımı göz önünde bulundurularak $f(R)$ gravitasyon teorisi çerçevesinde konformal düz uzay-zamanda bulunan toz bulutu için $f(R)$ fonksiyonu

$$
\begin{aligned}
f(R) & =\int f_{R} d R \\
& =6 c_{1} c_{3} \ln \left(R-12 c_{1} c_{2}\right)
\end{aligned}
$$

şeklinde elde edilir.

\section{Tartışma ve Sonuçlar}

Toz bulutu ile dolu konformal düz uzay-zamana ait alan denklemleri ve tam çözümleri $f(R)$ gravitasyon teorisi çerçevesinde elde edildi. (16) denkleminden görüldügü üzere toz bulutunun enerji yoğunluğu zamandan bağımsız sabit bir değer almaktadır. Herhangi bir kozmik maddenin fiziksel olarak anlamlı olabilmesi için pozitif enerji yoğunluğuna sahip olması gerektiği yaygınca bilinen bir gerçektir. Bu nedenle (16) denklemindeki toz madde yoğunluğunun pozitif bir değer alabilmesi için keyfi sabitlerin $\quad c_{1} c_{3}<0 \quad$ koşulunu sağlaması gerekmektedir. (18) denklemi ile verilen Ricci skaleri için kritik bir radyal değer belirlenebilir. $\mathrm{Bu}$ değer $R\left(r_{k r i .}\right)=0$ olarak düşünülürse, $r_{k r i .}=\sqrt{\frac{3 c_{2}}{c_{1}}}$ olur. Dolayısıyla, $\quad r_{k r i .}>\sqrt{\frac{3 c_{2}}{c_{1}}}$ durumunda, elde edilen model için Ricci skaleri sıfırdan farklı bir değer almaktadır. Bu kritik değer elde edilen uzay-zamanın pozitif veya negatif eğrilikli olmasını doğrudan etkileyen değerdir. Toz bulutu bulunan konformal düz uzay-zamanın (14) denklemi ile verilen metrik potansiyelinin radyal koordinata bağlı olarak değişimi Şekil 1'de verilmiştir.

Şekil 1'de görüldüğü üzere metrik potansiyel, A(r), radyal koordinata bağlı olarak azalan bir fonksiyondur. 


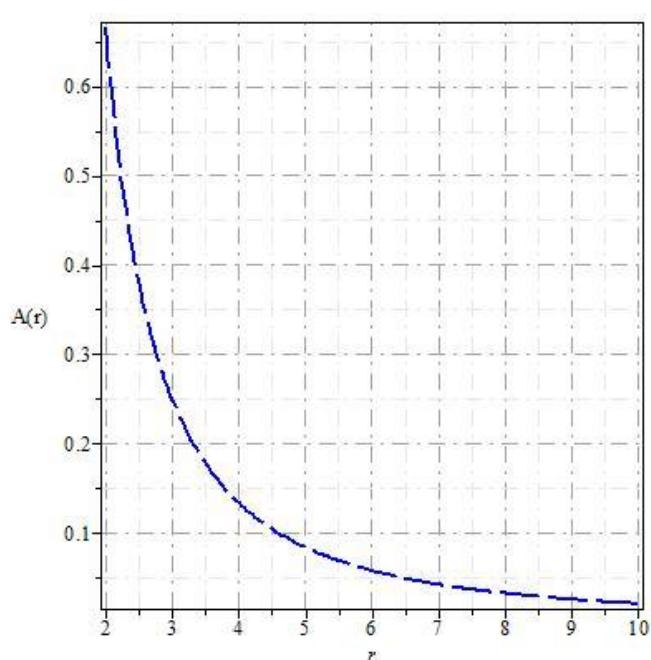

Şekil 1. $A(r)$, Metrik Potansiyelin Radyal Koordinata Göre Değişimi $\left(c_{1}=-1\right.$ ve $\left.c_{2}=1\right)$.

Limit durumları incelendiğinde, $r \rightarrow 0$ olduğu durumda, $c_{2}$ sabitinin işaretine bağlı olarak sabit değer alırken, $r \rightarrow \infty$ olduğu durumda sıfır olmaktadır. Ayrıca, (17) denklemi ile verilen toz bulutu bulunan statik konformal simetrik uzayzaman için kinematik nicelikler, sırasıyla genişleme skaleri, rotasyon tensörü ve shear tensörü

$$
\begin{gathered}
\theta=0, \\
\omega_{\mathrm{ik}}=0
\end{gathered}
$$

ve

$$
\sigma_{\mathrm{ik}}=0
$$

olarak elde edildi. Dolayısıyla elde edilen modelin statik bir yapıya sahip olduğu bu sonuçlar ile de desteklenmektedir. Diğer yandan, (21) denklemi ile verilen $f(R)$ fonksiyonu logaritmik terim içermektedir. Yapılan çalışmalarda eğri bir uzayzamandaki kuantum etkilerinin gravitasyonel Lagrangianda logaritmik terimlere sebep olabileceği düşünülmektedir [36]. Bu kapsamda logaritmik terim içeren birçok $f(R)$ fonksiyonu literatürde çalışılmıştır. En genel olarak, Capozziello ve ark. [37] tarafindan

$$
f(R)=\alpha \ln R
$$

modeli önerilmiştir. Burada $\alpha$, boyutsuzluk sabitidir. Bu modeli SNela Hubble diyagramı ve rahat gökada kümelerindeki gaz kütlesi fraksiyonu verileri ile karşılaştırmak için kullanmışlardır. (21) denkleminde $c_{2}$ sabiti sıfır olarak seçilirse toz bulutu bulunan konformal düz uzay-zaman için elde edilen $f(R)$ fonksiyonu (22) denklemi ile verilen modele karşılık gelmektedir. Bu durumda boyutsuzluk sabiti $\alpha=$ $6 c_{1} c_{3}$ değerini alır. Öyleyse söylenebilir ki; rahat gök ada kümelerindeki gaz kütlesi sabit varsayılır ve gök ada geometrisi konformal düz uzay-zaman geometrisine uygun seçilirse; bu çalışmada elde edilen toz bulutu ile dolu konformal düz uzay-zaman çözümleri rahat gök adalar için özel bir model kabul edilebilir.

\section{Teşekkür}

$\mathrm{Bu}$ çalışmaya değerli yorumları ile katkı sağlayan sayın Dr. Öğr. Üyesi Melis Ulu Doğru'ya çok teşekkür ederim.

\section{Kaynakça}

[1] Perlmutter, S., Aldering, G., Goldhaber, G., Knop, R. A., Nugent, P., Castro, P. G., Deustua, S., Fabbro, S., Goobar, A., Groom, D. E., Hook, I. M., Kim, A. G., Kim, M. Y., Lee, J. C., Nunes, N. J., Pain, R., Pennypacker, C. R., Quimby, R., Lidman, C., Ellis, R. S., Irwin, M., McMahon, R. G., RuizLapuente, P., Walton, N., Schaefer, B., Boyle, B. J., Filippenko, A. V., Matheson, T., Fruchter, A. S., Panagia, N., Newberg, H. J. M., Couch, W. J. 1999. Measurements of $\Omega$ and $\Lambda$ from 42 High-Redshift Supernovae. Astrophysical Journal, 517(1999), 565.

[2] Riess, A. G., Filippenko, A. V., Challis, P., Clocchiatti, A., Diercks, A., Garnavich, P. M., Gilliland, R. L., Hogan, C. J., Jha, S., Kirshner, R. P., Leibundgut, B., Phillips, M. M., Reiss, D., Schmidt, B. P., Schommer, R. A., Smith, R. C., Spyromilio, J., Stubbs, C., Suntzeff, N. B., Tonry, J. 1998. Observational Evidence from Supernovae for an Accelerating Universe and a Cosmological Constant. The Astronomical Journal, 116(1998), 1009.

[3] Astier, P., Guy, J., Regnault, N., Pain, R., Aubourg, E., Balam, D., Basa, S., Carlberg, R. G., Fabbro, S., Fouchez, D., Hook, I. M., Howell, D. A., Lafoux, H., Neill, J. D., Palanque-Delabrouille, N., Perrett, K., Pritchet, C. J., Rich, J., Sullivan, M., Taillet, R., Aldering, G., Antilogus, P., Arsenijevic, V., Balland, C., Baumont, S., Bronder, J., Courtois, H., Ellis, R. S., Filiol, M., Gon, calves, A. C., Goobar, A., Guide, D., Hardin, D., Lusset, V., Lidman, C., McMahon, R., Mouchet, M., Mourao, A., Perlmutter, S., Ripoche, P., Tao, C., Walton, N. 2006. The Supernova Legacy Survey: measurement of $\Omega_{m}, \Omega_{\Lambda}$ and $\mathrm{w}$ from the first year data set. Astronomy \& Astrophysics, 447(2006), 31-48.

[4] Spergel, D. N., Verde, L., Peiris, H. V., Komatsu, E., Nolta, M. R., Bennett, C. L., Halpern, M., Hinshaw, G., Jarosik, N., Kogut, A., Limon, M., Meyer, S. S., Page, L., Tucker, G. S., Weiland, J. L., Wollack, E., Wright, E. L. 2003. First Year Wilkinson Microwave Anisotropy Probe (WMAP) Observations: Determination of Cosmological Parameters. The Astrophysical Journal Supplement Series, 148(2003), 175.

[5] Bennett, C. L., Halpern, M., Hinshaw, G., Jarosik, N., Kogut, A., Limon, M., Meyer, S. S., Page, L., Spergel, D. N., Tucker, G. S., Wollack, E., Wright, E. L., Barnes, C., Greason, M. R., Hill, R. S., Komatsu, E., Nolta, M. R., Odegard, N., Peiris, H. V., Verde, L., Weiland, J. L. 2003. First Year 
Wilkinson Microwave Anisotropy Probe (WMAP) Observations: Preliminary Maps and Basic Results. The Astrophysical Journal Supplement Series, 148(2003), 1.

[6] Allen, S. W., Schmidt, R. W., Ebeling, H., Fabian, A. C., \& van Speybroeck, L. 2004. Constraints on dark energy from Chandra observations of the largest relaxed galaxy clusters. Monthly Notices of the Royal Astronomical Society, 353(2004), 457.

[7] Tegmark, M., Strauss, M. A., Blanton, M. R., Abazajian, K., Dodelson, S., Sandvik, H., Wang, X., Weinberg, D. H., Zehavi, I., Bahcall, N. A., Hoyle, F., Schlegel, D., Scoccimarro, R., Vogeley, M. S., Berlind, A., Budavari, T., Connolly, A., Eisenstein, D. J., Finkbeiner, D., Frieman, J. A., Gunn, J. E., Hui, L., Jain, B., Johnston, D., Kent, S., Lin, H., Nakajima, R., Nichol, R. C., Ostriker, J. P., Pope, A., Scranton, R., Seljak, U., Sheth, R. K., Stebbins, A., Szalay, A. S., Szapudi, I., Xu, Y., Annis, J., Brinkmann, J., Burles, S., Castander, F. J., Csabai, I., Loveday, J., Doi, M., Fukugita, M., Gillespie, B., Hennessy, G., Hogg, D. W., Ivezić, Ž., Knapp, G. R., Lamb, D. Q., Lee, B. C., Lupton, R. H., McKay, T. A., Kunszt, P., Munn, J. A., O'Connell, L., Peoples, J., Pier, J. R., Richmond, M., Rockosi, C., Schneider, D. P., Stoughton, C., Tucker, D. L., vanden Berk, D. E., Yanny, B., York, D. G. 2004. Cosmological parameters from SDSS and WMAP. Physical Review D, 69(2004), 103501.

[8] Shamir, M. F., Jhangeer, A., Bhatti, A. A. 2012. Conserved Quantities in $f(R)$ Gravity via Noether Symmetry. Chinese Physics Letters, 29(2012), 080402.

[9] Mohammadi, A. K., Malekjani M., Monshizadeh, M. 2012. Reconstruction Of Modified Gravity With Ghost Dark Energy Models. Modern Physics Letters A, 27(2012), 1250100.

[10] Martin, J. 2008. Quintessence: A Mini-Review. Modern Physics Letters A, 23(2008), 1252.

[11] Nojiri, S., Odintsov, S.D., Sami, M. 2006. Dark energy cosmology from higher-order, stringinspired gravity, and its reconstruction. Physical Review D, 74(2006), 046004.

[12] Padmanabhan, T., Chaudhury, T.R. 2002. Can the clustered dark matter and the smooth dark energy arise from the same scalar field?. Physical Review D, 66(2002), 081301.

[13] Chiba, T., Okabe, T., Yamaguchi, M. 2000. Kinetically driven quintessence. Physical Review D, 62(2000), 023511.

[14] Bento, M. C., Bertolami, O., Sen, A.A. 2002. Generalized Chaplgin gas, accelerated expansion, and dark-energy-matter unification. Physical Review D, 66(2002), 043507.

[15] Mishra, B., Tripathy, S. K., Tarai, S., 2018. Cosmological models with a hybrid scale factor in an extended gravity theory. Modern Physics Letter A, 33(2008), 1850052.

[16] Buchdahl, H. A. 1970. Non-linear Lagrangians and cosmological theory. Monthly Notices of the Royal Astronomical Society, 150(1970), 1.

[17] Bengochea, G. R., Ferraro, R. 2009. Dark torsion as the cosmic speed-up. Physical Review D, 79(2009), 124019.

[18] Harko, T., Lobo, F. S. N., Nojiri, S., Odintsov, S. D. 2011. f(R,T) gravity. Physical Review D, 84(2011), 024020.

[19] Bamba, K., Odintsov S. D., Sebastiani, L., Zerbini, S. 2010. Finite-time future singularities in modified Gauss-Bonnet and F(R,G) gravity and singularity avoidance. The European Physical Journal C, 67 (2010), 295.

[20] Nojiri, S., Odintsov, S. D. 2007. Introduction to Modified Gravity and Gravitational Alternative for Dark Energy. International Journal of Geometric Methods in Modern Physics. 4(2007), 115.

[21] Nojiri, S., Odintsov, S. D. 2008. Dark energy, inflation and dark matter from modified $\mathrm{F}(\mathrm{R})$ gravity. https://arxiv.org/pdf/0807.0685.pdf. (Erişim tarihi : 05.07.2008).

[22] Carroll, S.M., Duvvuri, V., Trodden, M., Turner, M.S. 2004. Is cosmic speed-up due to new gravitational physics?. Physical Review D, 70(2004), 043528.

[23] Rebouças, M. J., Santos, J. 2009. Gödel-Type Universes in $f(R)$ Gravity. Physical Review D, 80(2009)(6): 063009.

[24] Sharif, H., Shamir, M. F. 2009. Exact Solutions of Bianchi I and V Spacetimes in $f(R)$ Theory of Gravity. Classical and Quantum Gravity, 26(2009), 235020.

[25] Pradhan, A., Pandey, O. P. 2002. Conformally Flat Spherically Symmetric Cosmological Models-Revisited. Spacetime and Substance, 4(2002), 169-173.

[26] Reddy, D. R. K. 1979. Spherically symmetric static conformally flat solutions in Brans-Dicke and Sen-Dunn theories of gravitation. Journal of Mathematical Physics, 20(1979), 23.

[27] Reddy, D. R. K., Avadhanulu, M. B., Venkateswarlu, R. 1988. A Static ConformallyFlat Vacuum Model in Self-Creation Cosmology. Astrophysics and Space Science, 141(1988), 181-184.

[28] Yadav, R. B. S., Prasad, U. 1993. Non-static conformally flat spherically symmetric perfect fluid distributionin Einstein-Cartan theory. Astrophysics and Space Science, 203(1993), 37-42. 
[29] Khadekar, G. S., Nagpure, A. R. 2001. Higher Dimensional Static Cosmological Model in Lyra Manifold. https://arxiv.org/pdf/gr-qc/0111096 .pdf (Erişim tarihi : 28.11.2001).

[30] Abebe, G., Govinder K. S., Maharaj S. D. 2013. Lie symmetries for a conformally flat radiating star. International Journal of Theoretical Physics, 52(2013): 3244-3254.

[31] Sharif, M., Kausar, H. R. 2011. Dust Static Spherically Symmetric Solution in $f(R)$ Gravity. Journal of the Physical Society of Japan, 80(2011), 044004.

[32] Shamir, M. F., Raza, Z. 2014. Dust Static Cylindrically Symmetric Solutions in $f(R)$ Gravity. Communications in Theoretical Physics, 62(2014), 348-352.

[33] Shamir, M. F., Ahmad, Z., Raza, Z. 2015. Gravitational Dust Collapse in f(R) Gravity.
International Journal of Theoretical Physics, 54(2015), 1450-1460.

[34] Lobo, F. S. N., Oliveira, M. A. 2009. Wormhole geometries in $f(R)$ modified theories of gravity. Physical Review D, 80(2009), 104012.

[35] Gron, O., Johannesen, S. 2011. A solution of the Einstein-Maxwell equations describing conformally flat spacetime outside a charged domain wall. https://arxiv.org/pdf/1104.1383. pdf. (Erişim tarihi : 07.04.2011).

[36] Nojiri, S., Odintsov, S. D. 2004. Modified gravity with $\ln R$ terms and cosmic acceleration. General Relativity and Gravitation, 36(2004), 17651780.

[37] Capozziello, S., Cardone, V.F., Francaviglia, M. 2006. $f(R)$ theories of gravity in Palatini approach matched with observations. General Relativity and Gravitation, 38(2006), 711-734. 